\title{
COVID-19 IMPACT ON LIVER TRANSPLANTATION RECIPIENTS
}

\author{
Impacto da Covid-19 em recetores de transplante hepático
}

\author{
André Carmo', Cristiana Marques², Dulce Diogo ${ }^{3}$
}

\begin{abstract}
Introduction: Since the beginning of corona virus infectious disease (COVID-19) pandemic and the fact that liver transplantation (LT) recipients are immunosuppressed population, a discussion was started about the higher risk for contracting the disease: there was controversy of risk factors for the disease severity and mortality as to the baseline immunosuppression (IS) regimen and target therapy for COVID-19 management. Purpose: The present work intends to analyze publications (clinical cases and series) to conclude on demographic risk factors, baseline and management of the IS specific COVID-19 therapy and outcome in these patients. Materials and Methods: A research on MEDLINE and PubMed databases was conducted. A total of 127 articles were identified, and 55 included for the final quantitative analysis. Statistical analysis was conducted by using the chi-square test, spearman correlation, and logistic regression. Descriptive analysis were presented in number, percentage or mean and range. Results: A total of 111 single cases were analyzed (95 adults and 16 pediatric). Regarding the adult population, $66(69.47 \%)$ were male and the mean age was 58,73 years. The most common comorbidities were obesity/overweight (35.79\%), arterial hypertension (33.68\%), and diabetes (27.37\%). The most used immunosuppressant was tacrolimus (74.74\%) and mycophenolate mofetil (45.26\%). Forty-one patients (43.16\%) presented complications during treatment and $12(12.63 \%)$ required invasive ventilation. The mortality rate in the adult population was $20 \%$. Regarding the pediatric population, seven $(43.75 \%)$ were male and the mean age was 1.28 years. The most used immunosuppressant was tacrolimus $(93.75 \%)$. Only four patients $(25 \%)$ presented complications, and three $(18.75 \%)$ required invasive ventilation. Mortality among the pediatric population was $18.75 \%$. Discussion: Justifications were presented based on the literature for the main statistically significant associations. Whenever possible, comparisons were made with other works. Special emphasis was given to comorbidities and immunosuppression. Conclusion: Older age, diabetes, higher white blood cells and support ventilation were associated with worse outcome. Lymphopenia and higher C-reactive protein levels were associated with a severe course of the disease. Mycophenolate mofetil as a baseline immunosuppression regimen was associated with shock during hospital stay. Overall, the mortality rate was higher in LT recipients than in the general population.
\end{abstract}

Keywords: Liver transplantation; COVID-19; Postoperative Complications.

\footnotetext{
Institution:

${ }^{1}$ Faculty of Medicine, University of Coimbra, University of Coimbra, Coimbra, Portugal

${ }^{2}$ Faculty of Psychology and Educational Sciences, Center for Research in Neuropsychology and Cognitive Behavioral Intervention (CINEICC), University of Coimbra, Coimbra, Portugal

${ }^{3}$ Adult Liver Transplantation Unit, Surgery Department, Coimbra Hospital and Universitary Center, Coimbra, Portugal
}

Correspondence:

Dulce Diogo

dulce.diogo@chuc.min-saude.pt

Recebido em: 22/03/2021

Aceito em:26/04/2021

\section{INTRODUCTION}

What was considered to be a "viral pneumonia" in Wuhan, China, evolved to a PHEIC, and on March 11, 2020 , it was considered a pandemic by the WHO. ${ }^{1}$ The etiological agent responsible was identified as severe acute respiratory syndrome coronavirus, ${ }^{2}$ responsible for coronavirus infectious disease (COVID-19). Until October 7, 2020, there have been reported above 76 million COVID-19 cases, and above 1,7 million deaths in 235 countries, areas or territories. ${ }^{2}$ 
The first case of an infection by SARS-CoV-2 in Portugal was confirmed on March 2, $2020^{3}$ and, on March 18, 2020, the Emergency State was declared for the first time in modern democracy. ${ }^{4}$ To date, there are 383258 confirmed cases and 6413 deaths by COVID-19 ${ }^{5}$ in Portugal.

Many risk factors were associated with a severe SARSCoV-2 infection in liver transplant (LT) recipients. Those are elderly patients (over 60 years old), male gender and one or more comorbidities (such as hypertension, diabetes, COPD, and malignant tumors). ${ }^{6-11}$ Also, some laboratory results have been independently associated with poor prognosis, being lymphopenia, ${ }^{12,13}$ elevated CRP, ${ }^{11}$ a high SOFA score and high d-dimers at admission. $^{7}$

Although there is some agreement on the risk factor for disease severity and poor prognosis, when talking about baseline and IS management there is still no consensus. On one hand, some authors defend that higher doses of immunosuppressants are at an elevated risk for the severe disease,${ }^{14}$ and, on the other hand, others present that long term LT recipients on low IS doses, results in prolonged shedding, and consequently, an increase of viral loads and severe clinical presentation, not directly related to the IS. ${ }^{15,16}$ Moreover, some physicians showed that $\mathrm{LT}$ recipients are not at a higher risk of contracting COVID-19 ${ }^{17}$ when compared to the general population, while others presented that LT recipients are at higher risk. ${ }^{18}$

Until this moment, there is no specific therapy for COVID-19. The first drugs used were HCQ and Lopinavir/ Ritonavir. Recent studies showed that both therapies are ineffective, and should not be used in the treatment neither in the prevention of SARS-COV-2 infection. ${ }^{19}$ Moreover, $\mathrm{HCQ}$ has important drug interactions with cyclosporine, TAC and SIR, and lopinavir/ritonavir with SIR, EVE, TAC and cyclosporine, requiring close surveillance of these drug levels. ${ }^{20}$ Tocilizumab has shown promising results in reducing mortality of ventilated patients or in patients with severe disease, however, phase III studies have not demonstrated the efficacy of such IL-6 inhibitor. ${ }^{19}$ Other potential pharmacological agents in study are remdesivir, and convalescent plasma.

Due to this urgent need for orientation, we conduct a study on all clinical cases in the literature until December 23, 2020 analyzing the age, post livertransplantation time, comorbidities, baseline IS regimen, main laboratory findings, IS management during treatment, other pharmacological and COVID-19 treatments, complications during treatment, support ventilation, and outcome of all these patients.

\section{MATERIALS AND METHODS}

\section{Study Identification}

A search within MEDLINE and PubMed databases (https://pubmed.ncbi.nlm.nih.gov) was conducted using MeSH terms: "COVID-19" (related MeSH terms are: 2019 novel coronavirus, SARS-CoV-2 infection, 2019-nCoV infection) AND "liver transplantation" AND "recipient" from September $3^{\text {rd }}$ to $23^{\text {rd }}$, December 2020. The database used for this systematic review was MEDLINEPubMed. All terms used on the PubMed search were developed according to the PICO structure: population, intervention, comparison/control and outcome (PICO). Population: children and adults who were liver transplant recipients and diagnosed with COVID-19. Intervention: children and adults who were liver transplant recipients and diagnosed with COVID-19 that presented complications during treatment, died or were discharged home. Control/Comparison: all cases were compared as to comorbidities, laboratory findings, therapy received, and outcome. Outcome: medical management regarding immunosuppression, infectious therapy, established guidelines or consensus, and survival of COVID-19 liver recipients.

\section{Study Selection}

Case reports, original articles, reviews, correspondence, letters to editor, clinical randomized controlled trials, non-randomized controlled trials, consensus articles, and protocol studies were included. Papers published in languages other than English were translated using the Google Translate tool.

Studies on organs other than liver or with data impossible to differentiate from the liver transplantation, epidemiological studies, surveys, those on clinical presentation of COVID-19, and those unrelated to liver transplantation were excluded from this meta-analysis. Figure 1 presents a flow diagram of the research based on the Preferred Reporting Items for Systematic Reviews and Meta-Analysis (PRISMA). ${ }^{21}$

\section{Statistical analysis}

Statistical analysis was performed by using IBM SPSS 25 software.

Descriptive analysis was presented in number and percentage or mean, standard deviation and range.

Chi-square tests were used to test the independence between categorical variables (association between initial symptoms, baseline and IS management, other pharmacological therapy and support ventilation, with complications and outcome). Spearman rank order correlations (rho) were used to calculate the strength 
Figure 1. PRISMA flow diagram for the research.
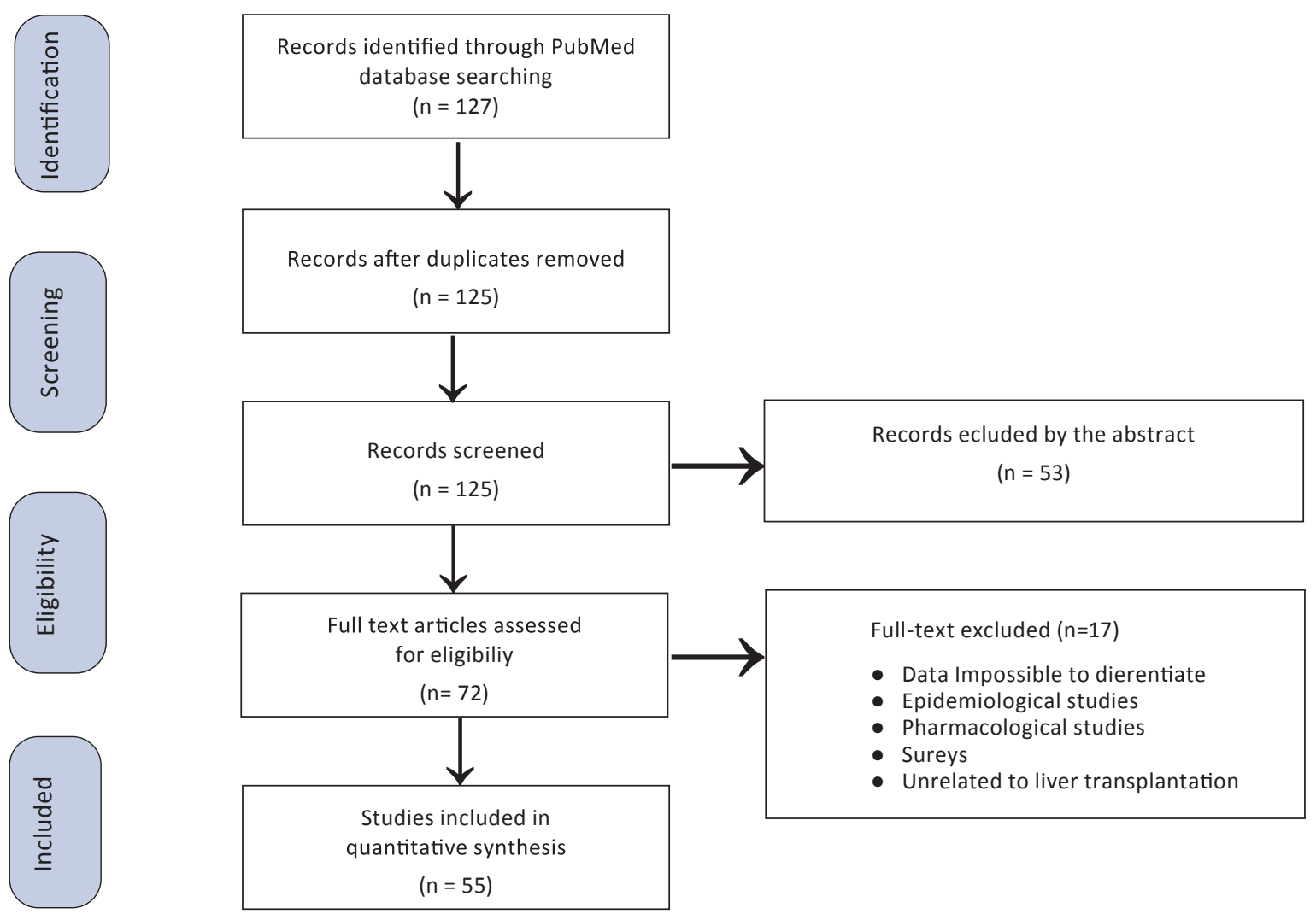

of the relationship between age and complications, age and outcome, time after LT and complications, and time after LT and outcome. Logistic regressions were used to test if comorbidities and main laboratory findings have an effect on the presence of complications (yes/no) and outcome (alive/dead) variables. A 95\% confidence interval was established.

For all the analyses, a value of $p<.05$ was considered significant.

\section{RESULTS}

A total of 55 articles were analyzed. Regarding those articles, 111 single cases were reviewed, in which 95 $(85.59 \%)$ were adult patients and the remaining 16 $(14,41 \%)$ were pediatric.

\section{Adult population}

Regarding the adult population, $66(69,47 \%)$ were male, $27(28.42 \%)$ female, and $2(2.11 \%)$ cases have not been specified. The mean age was 58.73 years ( $\sigma=13,57$ years), ranging between 18 and 85 years. The distribution of the population by age and gender is represented in Figure 2. Four (4.21\%) patients were white, 1 (1.05\%) black, 1 (1.05\%) Asian, 1 (1.05\%) Indian, 1 (1.05\%) Hispanic, 2 African American (2.11\%), and 85 $(89.47 \%)$ have not been specified. The mean time from liver transplantation to COVID-19 diagnosis was 6.98 years ( $\sigma=7.129$ years), ranging from 0 to 28.2 years. In one case, the time between transplantation to COVID-19 diagnosis have not been specified.

The most common cause for liver transplantation was hepatocellular carcinoma $(n=20 ; 21.05 \%)$ and HCV infection ( $n=20 ; 21.05 \%$ ), followed by hepatitis $B$ virus infection ( $n=14 ; 14.74 \%$ ), alcoholic cirrhosis ( $n=10$; $10.53 \%)$, and PSC ( $n=4 ; 4.21 \%)$. The most common comorbidities were overweight/obesity ( $n=34 ; 35.79 \%)$, arterial hypertension ( $n=32 ; 33.68 \%$ ), diabetes mellitus $(n=26 ; 27.37 \%)$, cardiovascular disease $(n=15 ; 15.79 \%)$, and chronic kidney disease $(n=14 ; 14.74 \%)$. In $19(20 \%)$ cases there were no comorbidities. The most frequent maintained immunosuppression regimen in monotherapy or in combination was TAC ( $\mathrm{n}=71 ; 74.74 \%)$, MMF ( $\mathrm{n}$ $=43 ; 45.26 \%)$, prednisone $(n=21 ; 22.11 \%)$, EVE $(n$ $=7 ; 7.37 \%$ ), and methylprednisolone $(\mathrm{n}=7 ; 7.37 \%)$. All demographic data are presented in Table 1. 
The most prevalent initial symptoms between the adult population were fever $(n=63 ; 66.32 \%)$, cough $(n=38$; $40 \%)$, shortness of breath $(n=33 ; 34.74 \%)$ and diarrhea $(n=20 ; 21.05 \%)$. The mean time from the initial symptoms to hospital admission was $4.35(\sigma=6.31)$ days, ranging from 0 to 40 days. In 38 (40\%) cases the time between initial symptoms to hospital admission have not been specified.

Figure 2. Distribution of the adult population by age and sex

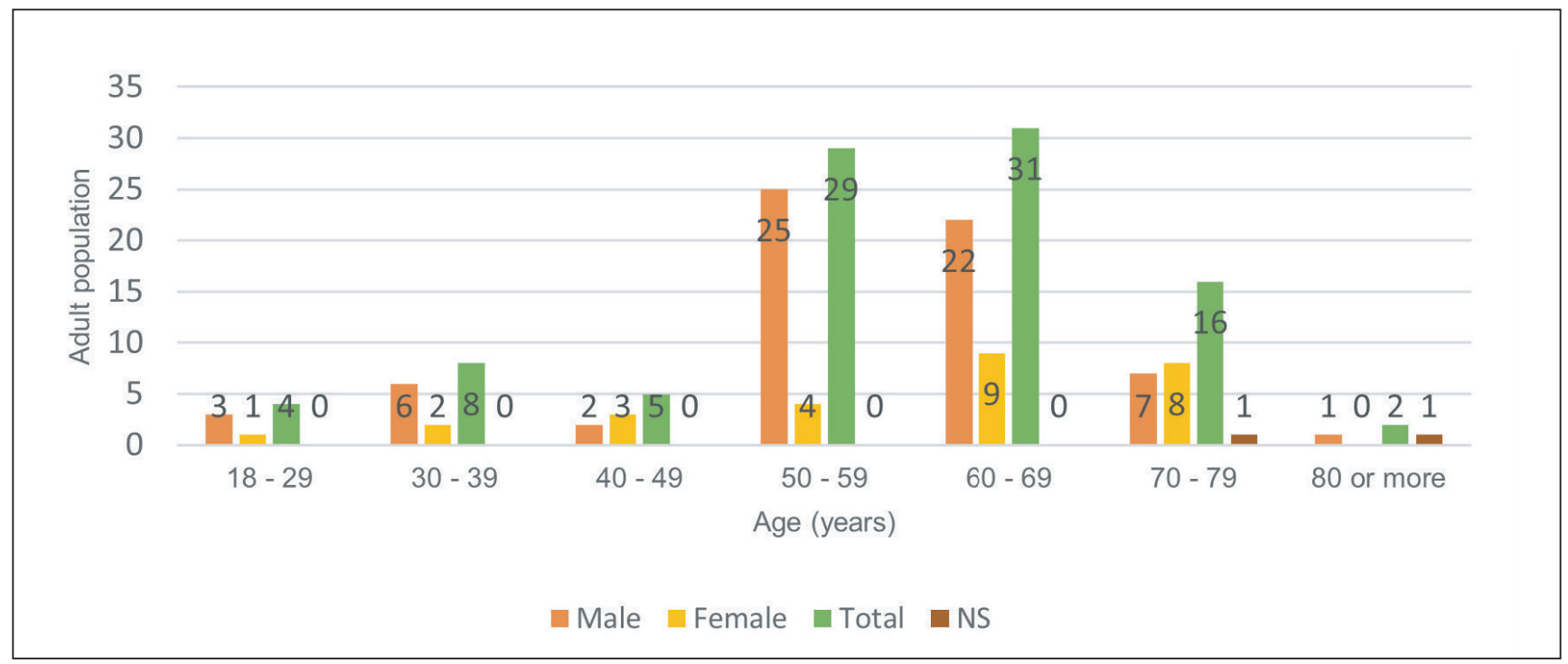

All laboratory findings correspond to the peak of the disease. SpO2 varied between 50 and $100 \%$ (mean of $87.36 \%$; $\sigma=10.30 \%$ ), hemoglobin from 6.6 to $12.4 \mathrm{~g} / \mathrm{dL}$ (mean of $9.81 \mathrm{~g} / \mathrm{dL} ; \sigma=2.12 \mathrm{~g} / \mathrm{dL}$ ), WBC between 0.66 and $45.9 \times 109 \mathrm{cells} / \mathrm{L}$ (mean of $7.50 \times 10^{9} \mathrm{cells} / \mathrm{L} ; \sigma=8.22$ $\left.\mathrm{x} 10^{9} \mathrm{cells} / \mathrm{L}\right)$, lymphocytes from 0.04 to $2.5 \times 10^{9} \mathrm{cells} / \mathrm{L}$ (mean of $0.68 \times 10^{9} \mathrm{cells} / \mathrm{L} ; \sigma=0.46 \times 10^{9} \mathrm{cells} / \mathrm{L}$ ), platelets from 20000 to 310000 cells/ $\mu \mathrm{L}$ (mean of 101346 cells/ $\mu \mathrm{L} ; \sigma=84805,64$ cells $/ \mu \mathrm{L}$ ), ALT between 8 and 634 $\mathrm{U} / \mathrm{L}$ (mean of $87.04 \mathrm{U} / \mathrm{L} ; \sigma=120.27 \mathrm{U} / \mathrm{L}$ ), AST from 16 to $770 \mathrm{U} / \mathrm{L}$ (mean of $78.63 \mathrm{U} / \mathrm{L} ; \sigma=117.09 \mathrm{U} / \mathrm{L}$ ), GGT between 24 and $3211 \mathrm{U} / \mathrm{L}$ (mean of $580.62 \mathrm{U} / \mathrm{L} ; \sigma=$ $846.17 \mathrm{U} / \mathrm{L}$ ), tBRB from 0.07 to $5.38 \mathrm{mg} / \mathrm{dL}$ (mean of $1.71 \mathrm{mg} / \mathrm{dL} ; \sigma=1.76 \mathrm{mg} / \mathrm{dL}$ ), CRP between 0.56 and $106.04 \mathrm{mg} / \mathrm{dL}$ (mean of $11.57 \mathrm{mg} / \mathrm{dL} ; \sigma=16.61 \mathrm{mg} / \mathrm{dL}$ ), IL-6 from 5 to $3385,06 \mathrm{pg} / \mathrm{mL}$ (mean of $665.91 \mathrm{pg} / \mathrm{dL}$; $\sigma=1130.69 \mathrm{pg} / \mathrm{dL}$ ), and ferritin form 18 to $2909 \mathrm{ng} / \mathrm{mL}$ (mean of $1153.95 \mathrm{ng} / \mathrm{mL} ; \sigma=896.28 \mathrm{ng} / \mathrm{mL}$ ). Sixteen (16.84\%) patients were admitted to the ICU. IS was lowered in $13(13.68 \%)$ cases, withdrawn or stopped in $24(25.26 \%)$ cases, added in five (5.26\%) patients, not changed in $40(42.11 \%)$ patients, and in $19(20 \%)$ cases the IS management have not been specified. Also, MMF dose was lowered in four $(4.21 \%)$ cases and withdrawn in $14(14.74 \%)$ cases.

$\mathrm{HCQ}$ was administered in 32 (33.68\%) patients, antiviral therapy in $16(16.84 \%)$ cases, antibiotics in 30 $(31.58 \%)$ cases, immunoglobulin in five $(5.26 \%)$ patients, and corticosteroids in four $(4.21 \%)$ cases. Invasive mechanical ventilation was used in 11 (11.58\%) patients, facial mask in five $(5.26 \%)$ patients, nasal cannula in 17 $(17.89 \%)$ cases, CPAP in three $(3.16 \%)$ cases, BiPAP in one $(1.05 \%)$ case, ECMO in one $(1.05 \%)$ patient, no support ventilation in $31(32.63 \%)$ cases, and ventilation was not specified in $22(23.16 \%)$ patients. Furthermore, 10 out of 11 patients $(90.91 \%)$ on mechanical ventilation died, two out of five patients (40\%) on facial mask also died and the same was observed in one case with CPAP and with ECMO.

During hospital stay, six (6.32\%) patients developed ARDS, seven $(7.37 \%)$ presented pneumonia, two (2.11\%) patients refractory shock, three $(3.16 \%)$ cases of graft dysfunction, four (4.21\%) patients developed acute kidney injury (AKI), $21(22.11 \%)$ patients did not develop any complication, and in 33 (34.74\%) cases complications have not been specified.

Regarding the outcome, 75 (78.95\%) patients were alive, $19(20 \%)$ patients died, and in one (1.05\%) case the outcome have not been specified. The distribution of death among the adult population by age and gender is represented in Figure 3 . The mean time of hospital stay/follow-up was 21.7 days ( $\sigma=16.18$ days), ranging from 1 to 75 days. In 38 $(40 \%)$ cases, the time of hospital stay/follow-up have not been specified. Table 1 presents a summary of each clinical case. 
Table 1. Summary fo the clinical cases used forthe adult population

\begin{tabular}{|c|c|c|c|c|c|c|}
\hline Reference & Country & Population & Baseline IS (cases) & $\begin{array}{l}\text { Main laboratory analyses } \\
\text { (mean) }\end{array}$ & IS management (cases) & Outcome \\
\hline Fernández-Ruiz et al ${ }^{22}$ & Spain & $\begin{array}{l}3 \text { males } \\
3 \text { females }\end{array}$ & $\begin{array}{c}\text { EVE (3) } \\
\text { TAC (2) } \\
\text { MMF (2) } \\
\text { Prednisone (1) } \\
\text { AZA (1) }\end{array}$ & $\begin{array}{c}\text { Lymphocytes } 1.05 \times 10^{\wedge} \mathrm{g} / \mathrm{L} \\
\text { ALT } 85.17 \mathrm{U} / \mathrm{L} \\
\text { CRP } 9.78 \mathrm{mg} / \mathrm{dL}\end{array}$ & $\begin{array}{l}\text { Stopped EVE (2) } \\
\text { Lower TAC dose (2) } \\
\text { Stopped MMF (2) } \\
\text { Added MMF (1) } \\
\text { Added TAC (2) } \\
\text { No change (1) }\end{array}$ & $\begin{array}{l}4 \text { alive } \\
2 \text { dead }\end{array}$ \\
\hline $\begin{array}{l}\text { Zhong Z et al. }{ }^{23} \\
\text { Qin J et al. }{ }^{24}\end{array}$ & China & 1 male & $\begin{array}{c}\text { TAC } \\
\text { Methylprednisolone }\end{array}$ & $\begin{array}{c}\text { Lymphocytes } 0.48 \times 10^{\wedge} 9 / \mathrm{L} \\
\text { Platelets } 74000 / \mu \mathrm{L} \\
\text { ALT } 424 \mathrm{U} / \mathrm{L} \\
\text { CRP } 10.18 \mathrm{mg} / \mathrm{dL}\end{array}$ & $\begin{array}{c}\text { Stopped TAC } \\
\text { Lower } \\
\text { methylprednisolone } \\
\text { dose }\end{array}$ & Alive \\
\hline Huang JF et al. ${ }^{25}$ & China & 1 male & $\begin{array}{l}\text { TAC } \\
\text { MMF }\end{array}$ & $\begin{array}{c}\text { Lymphocytes <0.1 x10^9/L } \\
\text { ALT } 60 \mathrm{U} / \mathrm{L} \\
\text { CRP } 3.51 \mathrm{mg} / \mathrm{dL}\end{array}$ & $\begin{array}{l}\text { Lower TAC dose } \\
\text { Lower MMF dose }\end{array}$ & Dead \\
\hline Liu B et al. ${ }^{26}$ & China & 1 male & TAC & $\begin{array}{c}\text { Lymphocytes } 0.42 \times 10^{\wedge} 9 / \mathrm{L} \\
\text { WBC } 5.9 \times 10^{\wedge} 9 / \mathrm{L} \\
\text { CRP } 3.21 \mathrm{mg} / \mathrm{dL}\end{array}$ & $\begin{array}{c}\text { Stopped TAC } \\
\text { Added } \\
\text { methylprednisolone } \\
\text { dose }\end{array}$ & Alive \\
\hline Hammami MB et al. ${ }^{27}$ & USA & 1 male & TAC & $\begin{array}{c}\text { Lymphocytes } 0.65 \times 10^{\wedge} 9 / \mathrm{L} \\
\text { Platelets } 49000 / \mu \mathrm{L} \\
\text { ALT } 27 \mathrm{U} / \mathrm{L} \\
\text { CRP } 1.49 \mathrm{mg} / \mathrm{dL}\end{array}$ & No change & Alive \\
\hline Lee BT et al. ${ }^{28}$ & USA & $\begin{array}{l}26 \text { males } \\
12 \text { females }\end{array}$ & NS & $\begin{array}{l}\text { Only related to the deaths: } \\
\text { Lymphocytes } 0.64 \times 10^{\wedge} 9 / \mathrm{L} \\
\text { ALT } 59.57 \mathrm{U} / \mathrm{L} \\
\text { CRP } 13.87 \mathrm{mg} / \mathrm{dL}\end{array}$ & NS & $\begin{array}{l}31 \text { alive } \\
7 \text { dead }\end{array}$ \\
\hline Hoek RAS et al. ${ }^{29}$ & Netherlands & 1 male & TAC & NS & No change & Alive \\
\hline Kates OS et al. ${ }^{30}$ & USA & 1 male & Cyclosporine & $\begin{array}{c}\text { Lymphocytes } 0.9 \times 10^{\wedge} 9 / \mathrm{L} \\
\text { WBC } 1.93 \times 10^{\wedge} 9 / \mathrm{L} \\
\text { ALT } 12 \mathrm{U} / \mathrm{L}\end{array}$ & No change & Alive \\
\hline Gao F et al. ${ }^{31}$ & China & 3 males & $\begin{array}{c}\text { TAC (3) } \\
\text { MMF (1) } \\
\text { Corticosteroids (1) }\end{array}$ & Lymphocytes 0.59 x10^9/L & $\begin{array}{l}\text { Lower TAC dose (1) } \\
\text { Lower corticosteroids } \\
\text { dose (1) } \\
\text { No change (1) } \\
\text { IS withdrawal (1) }\end{array}$ & $\begin{array}{l}2 \text { alive } \\
1 \text { dead }\end{array}$ \\
\hline Verma A et al. ${ }^{32}$ & UK & 5 males & $\begin{array}{l}\text { TAC (4) } \\
\text { Prednisone (4) } \\
\text { MMF (1) } \\
\text { AZA (1) }\end{array}$ & $\begin{array}{l}\text { Lymphocytes } 0.61 \times 10^{\wedge} 9 / \mathrm{L} \\
\text { WBC } 4.19 \times 10^{\wedge} 9 / \mathrm{L}\end{array}$ & $\begin{array}{l}\text { No change (4) } \\
\text { Lower MMF dose (1) } \\
\text { Higher prednisone } \\
\text { dose (1) }\end{array}$ & 5 alive \\
\hline Fung $\mathrm{M}$ et al..$^{33}$ & USA & 1 female & $\begin{array}{l}\text { TAC } \\
\text { MMF }\end{array}$ & $\begin{array}{c}\text { Lymphocytes } 0.54 \times 10^{\wedge} 9 / \mathrm{L} \\
\text { WBC } 4.9 \times 10^{\wedge} 9 / \mathrm{L} \\
\text { Platelets } 103000 / \mu \mathrm{L} \\
\text { ALT } 14 \mathrm{U} / \mathrm{L}\end{array}$ & No change & Alive \\
\hline Muller $\mathrm{H}$ et al. ${ }^{34}$ & Austria & 1 male & $\begin{array}{l}\text { TAC } \\
\text { MMF }\end{array}$ & $\begin{array}{l}\text { Normal WBC } \\
\text { CRP } 6.1 \mathrm{mg} / \mathrm{dL}\end{array}$ & No change & Alive \\
\hline Patrono D et al..$^{35}$ & Italy & $\begin{array}{c}8 \text { males } \\
2 \text { females }\end{array}$ & $\begin{array}{c}\text { TAC (10) } \\
\text { MMF (5) } \\
\text { Prednisone (3) } \\
\text { EVE (2) } \\
\text { MPA (1) }\end{array}$ & $\begin{array}{c}\text { Lymphocytes } 0.71 \times 10^{\wedge} 9 / \mathrm{L} \\
\text { WBC } 6.68 \times 10^{\wedge} 9 / \mathrm{L} \\
\text { ALT } 104 \mathrm{U} / \mathrm{L} \\
\text { CRP } 6.19 \mathrm{mg} / \mathrm{dL}\end{array}$ & $\begin{array}{c}\text { Stopped TAC (3) } \\
\text { No change (3) } \\
\text { Stopped MMF (1) } \\
\text { Lower MMF dose (1) } \\
\text { Lower TAC dose (1) } \\
\text { Higher prednisone } \\
\text { dose (1) } \\
\text { IS withdrawal (1) }\end{array}$ & $\begin{array}{l}8 \text { alive } \\
2 \text { dead }\end{array}$ \\
\hline
\end{tabular}


Covid-19 impact on liver transplantation recipients

Table 1. Summary fo the clinical cases used forthe adult population (cont.)

\begin{tabular}{|c|c|c|c|c|c|c|}
\hline Reference & Country & Population & Baseline IS (cases) & $\begin{array}{l}\text { Main laboratory analyses } \\
\text { (mean) }\end{array}$ & IS management (cases) & Outcome \\
\hline Hann A et al. ${ }^{36}$ & UK & $\begin{array}{l}2 \text { males } \\
1 \text { female }\end{array}$ & $\begin{array}{c}\text { TAC (3) } \\
\text { AZA (3) } \\
\text { Prednisone (2) }\end{array}$ & NS & NS & $\begin{array}{l}2 \text { alive } \\
1 \text { dead }\end{array}$ \\
\hline Donato MF et al. ${ }^{37}$ & Italy & $\begin{array}{c}6 \text { males } \\
2 \text { females }\end{array}$ & $\begin{array}{c}\text { TAC (7) } \\
\text { MMF (6) } \\
\text { Prednisone (2) }\end{array}$ & NS & NS & 8 alive \\
\hline Mehta SA et al. ${ }^{38}$ & USA & $\begin{array}{l}2 \text { males } \\
1 \text { female }\end{array}$ & $\begin{array}{c}\text { TAC (3) } \\
\text { MMF (2) } \\
\text { Prednisone (2) }\end{array}$ & $\begin{array}{l}\text { Lymphocytes } 0.60 \times 10^{\wedge} 9 / \mathrm{L} \\
\text { WBC } 3.13 \times 10^{\wedge} 9 / \mathrm{L} \\
\text { CRP } 20.83 \mathrm{mg} / \mathrm{dL}\end{array}$ & $\begin{array}{c}\text { No change (2) } \\
\text { Stopped TAC (1) } \\
\text { Stopped MMF (1) }\end{array}$ & 3 alive \\
\hline Prieto $\mathrm{M}$ et al. ${ }^{39}$ & Italy & 1 male & $\begin{array}{c}\text { MMF } \\
\text { Prednisone }\end{array}$ & $\begin{array}{c}\text { Lymphocytes } 0.2 \times 10^{\wedge} 9 / \mathrm{L} \\
\text { WBC } 4.9 \times 10^{\wedge} 9 / \mathrm{L} \\
\text { Platelets } 48000 / \mu \mathrm{L} \\
\text { ALT } 634 \mathrm{U} / \mathrm{L} \\
\text { Ferritin } 783 \mathrm{ng} / \mathrm{mL}\end{array}$ & Added TAC & Alive \\
\hline Jamir I et al. ${ }^{40}$ & India & 1 male & $\begin{array}{c}\text { TAC } \\
\text { MMF } \\
\text { Methylprednisolone }\end{array}$ & $\begin{array}{c}\text { Lymphocytes } 0.11 \times 10^{\wedge} 9 / \mathrm{L} \\
\text { WBC } 6.67 \times 10^{\wedge} 9 / \mathrm{L} \\
\text { Platelets } 31114 / \mu \mathrm{L} \\
\text { ALT } 405 \mathrm{U} / \mathrm{L} \\
\text { CRP } 106.04 \mathrm{mg} / \mathrm{dL}\end{array}$ & Stopped MMF & Alive \\
\hline Eslami P et al. ${ }^{41}$ & Iran & 1 male & $\begin{array}{c}\text { TAC } \\
\text { Methylprednisolone }\end{array}$ & $\begin{array}{l}\text { Lymphocytopenia } \\
\text { WBC } 15.7 \times 10^{\wedge} 9 / \mathrm{L} \\
\text { Platelets } 20000 / \mu \mathrm{L} \\
\text { ALT } 110 \mathrm{U} / \mathrm{L}\end{array}$ & NS & Dead \\
\hline García-Juaréz I et al. ${ }^{42}$ & Mexico & 1 female & TAC & $\begin{array}{c}\text { Lymphocytes } 0.87 \times 10^{\wedge} 9 / \mathrm{L} \\
\text { WBC } 2.8 \times 10^{\wedge} 9 / \mathrm{L} \\
\text { Platelets } 115000 / \mu \mathrm{L} \\
\text { ALT } 13 \mathrm{U} / \mathrm{L} \\
\text { CRP } 3.9 \mathrm{mg} / \mathrm{dL}\end{array}$ & Lower TAC dose & Alive \\
\hline Hatami B et al. ${ }^{43}$ & Iran & 1 female & $\begin{array}{c}\text { TAC } \\
\text { MMF } \\
\text { Prednisone }\end{array}$ & $\begin{array}{c}\text { WBC } 10.1 \times 10^{\wedge 9} / \mathrm{L} \\
\text { Platelets } 310000 / \mu \mathrm{L} \\
\text { ALT } 15 \mathrm{U} / \mathrm{L} \\
\text { CRP } 1.8 \mathrm{mg} / \mathrm{dL}\end{array}$ & Stopped MMF & NS \\
\hline Bosch F et al. ${ }^{44}$ & Germany & $\begin{array}{l}1 \text { male } \\
1 \text { female }\end{array}$ & $\begin{array}{l}\operatorname{MMF}(2) \\
\operatorname{EVE}(1)\end{array}$ & NS & $\begin{array}{l}\text { Stopped MMF (1) } \\
\text { No change (1) }\end{array}$ & 2 alive \\
\hline Antony SJ et al. ${ }^{45}$ & USA & 1 male & $\begin{array}{l}\text { TAC } \\
\text { Prednisone } \\
\text { MPA }\end{array}$ & $\begin{array}{c}\text { Lymphocytes } 0.2 \times 10^{\wedge} 9 / \mathrm{L} \\
\text { WBC } 12.13 \times 10^{\wedge} 9 / \mathrm{L} \\
\text { ALT } 64 \mathrm{U} / \mathrm{L} \\
\text { CRP } 3.7 \mathrm{mg} / \mathrm{dL}\end{array}$ & $\begin{array}{l}\text { IS withdrawal } \\
\text { Added } \\
\text { methylprednisolone }\end{array}$ & Alive \\
\hline Loinaz C et al. ${ }^{46}$ & Spain & $\begin{array}{l}11 \text { males } \\
2 \text { females }\end{array}$ & $\begin{array}{l}\text { MMF (7) } \\
\text { TAC (6) } \\
\text { Prednisone (2) } \\
\text { EVE (1) } \\
\text { MPA (1) }\end{array}$ & $\begin{array}{c}\text { Lymphocytes } 0.72 \times 10^{\wedge} 9 / \mathrm{L} \\
\text { WBC } 4.54 \times 10^{\wedge} 9 / \mathrm{L} \\
\text { ALT } 36.38 \mathrm{U} / \mathrm{L} \\
\text { CRP } 9.67 \mathrm{mg} / \mathrm{dL}\end{array}$ & No change (13) & 13 alives \\
\hline Modi AR et al. ${ }^{47}$ & USA & 1 male & $\begin{array}{c}\text { TAC } \\
\text { MMF } \\
\text { Prednisone }\end{array}$ & $\begin{array}{c}\text { Lymphocytes } 1.22 \times 10^{\wedge} 9 / \mathrm{L} \\
\text { WBC } 7.41 \times 10^{\wedge} 9 / \mathrm{L} \\
\text { ALT } 87 \mathrm{U} / \mathrm{L} \\
\text { CRP } 2 \mathrm{mg} / \mathrm{dL}\end{array}$ & $\begin{array}{l}\text { Lower TAC dose } \\
\text { Stopped MMF }\end{array}$ & Alive \\
\hline Felldin $\mathrm{M}$ et al. ${ }^{48}$ & Sweden & $\begin{array}{c}1 \text { male } \\
7 \text { females }\end{array}$ & $\begin{array}{c}\text { TAC (8) } \\
\text { MMF (4) } \\
\text { Prednisone (3) } \\
\text { AZA (1) } \\
\text { Methotrexate (1) }\end{array}$ & NS & $\begin{array}{c}\text { No change (4) } \\
\text { Stopped MMF (2) } \\
\text { Lower MMF dose (1) } \\
\text { Stopped methotrexate } \\
\text { (1) }\end{array}$ & \\
\hline
\end{tabular}


Table 1. Summary fo the cliical cases used forthe adult population (cont.)

\begin{tabular}{|c|c|c|c|c|c|c|}
\hline Reference & Country & Population & Baseline IS (cases) & $\begin{array}{l}\text { Main laboratory analyses } \\
\text { (mean) }\end{array}$ & IS management (cases) & Outcome \\
\hline Kolonko A et al..$^{49}$ & Poland & 1 male & $\begin{array}{c}\text { TAC } \\
\text { MMF } \\
\text { Prednisone }\end{array}$ & $\begin{array}{l}\text { ALT } 55 \mathrm{U} / \mathrm{L} \\
\text { AST } 18 \mathrm{U} / \mathrm{L} \\
\text { GGT } 101 \mathrm{U} / \mathrm{L} \\
\text { Low CRP }\end{array}$ & $\begin{array}{l}\text { Stopped MMF } \\
\text { Lower prednisone } \\
\text { dose }\end{array}$ & Alive \\
\hline Sessa A et al. ${ }^{50}$ & Italy & 1 male & TAC & $\begin{array}{c}\text { Lymphocytes } 0.39 \times 10^{\wedge} 9 / \mathrm{L} \\
\text { Platelets } 122000 / \mu \mathrm{L} \\
\text { ALT } 28 \mathrm{U} / \mathrm{L} \\
\text { CRP } 11.23 \mathrm{mg} / \mathrm{dL}\end{array}$ & No change & Alive \\
\hline Waisberg DR et al. ${ }^{51}$ & Brazil & $\begin{array}{l}4 \text { males } \\
1 \text { female }\end{array}$ & $\begin{array}{l}\operatorname{TAC}(5) \\
\operatorname{MMF}(4)\end{array}$ & $\begin{array}{c}\text { Lymphocytes } 0.87 \times 10^{\wedge} 9 / \mathrm{L} \\
\text { WBC } 16.99 \times 10^{\wedge} 9 / \mathrm{L} \\
\text { ALT } 133.4 \mathrm{U} / \mathrm{L} \\
\text { CRP } 12.02 \mathrm{mg} / \mathrm{dL}\end{array}$ & $\begin{array}{l}\text { No change (2) } \\
\text { Stopped MMF (2) } \\
\text { Lower TAC dose (1) } \\
\text { IS withdrawal (1) }\end{array}$ & $\begin{array}{l}3 \text { alive } \\
2 \text { dead }\end{array}$ \\
\hline Mathiasen VD et al. ${ }^{52}$ & Denmark & 1 female & $\begin{array}{l}\text { TAC } \\
\text { MMF }\end{array}$ & $\begin{array}{c}\text { Lymphocytes } 1.11 \times 10^{\wedge} 9 / \mathrm{L} \\
\text { WBC } 5.1 \times 10^{\wedge} 9 / \mathrm{L} \\
\text { Normal ALT } \\
\text { CRP } 3.72 \mathrm{mg} / \mathrm{dL}\end{array}$ & No change & Alive \\
\hline Kutzler HL et al..$^{53}$ & USA & 2 cases & $\mathrm{TC}(2)$ & NS & No change & 2 alive \\
\hline Mocchegiani F et al. ${ }^{54}$ & Italy & 1 male & TAC & $\begin{array}{l}\text { ALT } 30 \mathrm{U} / \mathrm{L} \\
\text { AST } 84 \mathrm{U} / \mathrm{L}\end{array}$ & No change & Dead \\
\hline Goss MB et al. ${ }^{55}$ & USA & $\begin{array}{l}4 \text { males } \\
6 \text { females }\end{array}$ & $\begin{array}{l}\text { TAC (10) } \\
\text { Prednisone (4) } \\
\text { SIR (2) } \\
\operatorname{MMF~(2)~}\end{array}$ & $\begin{array}{c}\text { Normal lymphocytes levels } \\
\text { Low WBC in } 2 \text { cases }\end{array}$ & $\begin{array}{l}\text { No change (9) } \\
\text { Lower sirolimus dose } \\
(1) \\
\text { Lower TAC dose (1) }\end{array}$ & 10 alive \\
\hline Imam A et al. ${ }^{56}$ & Turkey & 3 males & $\begin{array}{c}\text { TAC (3) } \\
\text { Corticosteroids (3) }\end{array}$ & NS & NS & 3 dead \\
\hline Heinz $\mathrm{N}$ et al. ${ }^{57}$ & USA & 1 female & $\begin{array}{c}\text { TAC } \\
\text { MMF } \\
\text { Methylprednisolone }\end{array}$ & $\begin{array}{c}\text { Lymphocytes } 4.5 \times 10^{\wedge} 9 / \mathrm{L} \\
\text { WBC } 18 \times 10^{\wedge} 9 / \mathrm{L} \\
\text { ALT } 980 \mathrm{U} / \mathrm{L} \\
\text { CRP } 4.63 \mathrm{mg} / \mathrm{dL}\end{array}$ & $\begin{array}{c}\text { Stopped MMF } \\
\text { Lower } \\
\text { methylprednisolone } \\
\text { dose }\end{array}$ & Alive \\
\hline Lagana et al.59 & USA & 1 female & MMF & $\begin{array}{l}\text { ALT } 1253 \mathrm{U} / \mathrm{L} \\
\text { AST } 908 \mathrm{U} / \mathrm{L} \\
\text { GGT } 473 \mathrm{U} / \mathrm{L}\end{array}$ & IS withdrawal & Alive \\
\hline
\end{tabular}

*The bold line separates the adult data (above the line) from the pediatric data (below the line).

ALT: alanine aminotransferase; AST: aspartate aminotransferase; AZA: azathioprine; CRP: C-reactive protein; EVE: Everolimus; GGT: gamma-glutamyltransferase; IS: immunosuppression; MMF: mycophenolate mofetil; MPA: mycophenolic acid; NS: not specified; SIR: sirolimus; TAC: tacrolimus; UK: United Kingdom; USA: United States of America; WBC: white blood cells. 
Figure 3. Distribution of death in the adult population by age and sex.

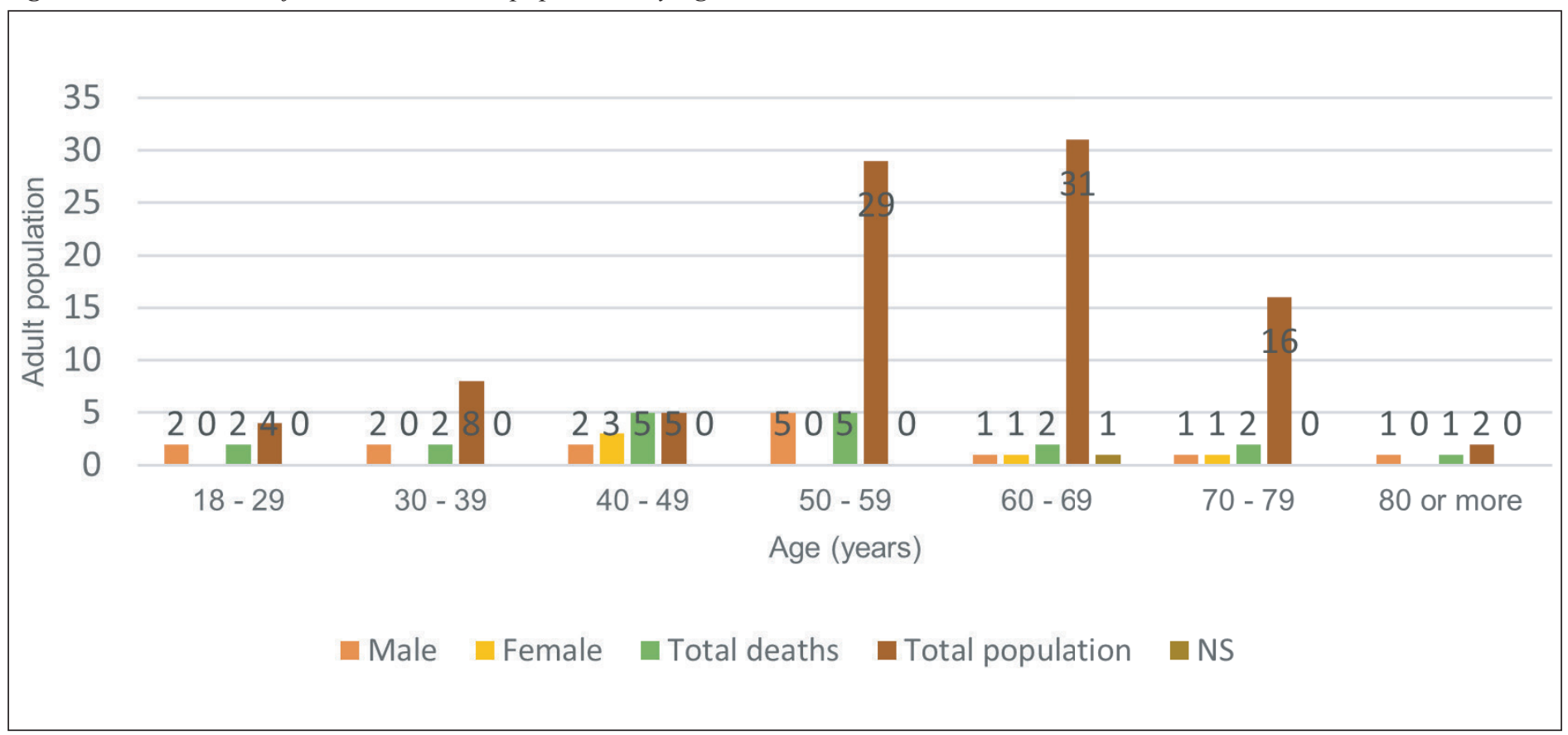

Table 2. Demographics for the study population

Adult Cases $(\mathrm{n}=95)$

n (\%)

Gender

Mean (SD)

NS

Ethnicity/Race

Male

Female

NS

Age

$\begin{array}{rcc}\text { White } & 4(4.21) & 4(25) \\ \text { Black } & 1(1.05) & 0(0) \\ \text { Asian } & 1(1.05) & 0(0) \\ \text { Hispanic } & 1(1.05) & 7(43.75) \\ \text { African American } & 2(2.11) & 0(0) \\ \text { Indian } & 1(1.05) & 0(0) \\ \text { NS } & 85(89.47) & 5(31.25)\end{array}$

Time after LT
27 (28.42)

$2(2.11)$

$58.73(13.57)$

$0(0)$

1.28 (1.49)

$10(62.5)$
Pediatric Cases ( $n=16$ )

$n$ (\%)

7 (43.75)

$9(56.25)$

$0(0)$
Mean (years)

NS
6.98

3.50

$1(1.05)$
$0(0)$ 
Table 2. Demographics for the study population (cont.)

\begin{tabular}{|c|c|c|}
\hline & Adult Cases (n=95) $n(\%)$ & Pediatric Cases (n=16) n (\%) \\
\hline \multicolumn{3}{|l|}{ Etiology for LT } \\
\hline $\mathrm{HCC}$ & $20(21.05)$ & $0(0)$ \\
\hline HCV infection & $20(21.05)$ & $0(0)$ \\
\hline HBV infection & $14(14.74)$ & $0(0)$ \\
\hline Alcoholic cirrhosis & $10(10.53)$ & $0(0)$ \\
\hline PSC & $4(4.21)$ & $0(0)$ \\
\hline Cryptogenic cirrhosis & $3(3.16)$ & $1(6.25)$ \\
\hline $\mathrm{NASH}$ & $3(3.16)$ & $0(0)$ \\
\hline ALF & $3(3.16)$ & $0(0)$ \\
\hline Al hepatitis & $2(2.11)$ & $0(0)$ \\
\hline Chronic rejection & $2(2.11)$ & $0(0)$ \\
\hline Budd-Chiari Syndrome & $2(2.11)$ & $0(0)$ \\
\hline Familial Amyloid Polyneuropathy & $1(1.05)$ & $0(0)$ \\
\hline Vasculitis & $1(1.05)$ & $0(0)$ \\
\hline Antiretroviral-induced hepatotoxicity & $1(1.05)$ & $0(0)$ \\
\hline Biliary atresia & $0(0)$ & $5(31.25)$ \\
\hline Hepatic adenomatosis & $0(0)$ & $1(6.25)$ \\
\hline Cholestatic liver disease & $0(0)$ & $1(6.25)$ \\
\hline NS & 32 (33.68) & $8(50)$ \\
\hline \multicolumn{3}{|l|}{ Comorbities } \\
\hline Overweigh/Obesity & 34 (35.79) & $3(18.75)$ \\
\hline AHT & $32(33.68)$ & $2(12.5)$ \\
\hline DM & $26(27.37)$ & $2(12.5)$ \\
\hline CVD & 15 (15.79) & $0(0)$ \\
\hline CKD & $14(14.74)$ & $1(6.25)$ \\
\hline Lung disease & $9(9.47)$ & $1(6.25)$ \\
\hline HIV & $5(5.26)$ & $0(0)$ \\
\hline Others & $27(28.42)$ & $12(75)$ \\
\hline None & $19(20)$ & $5(31.25)$ \\
\hline NS & 18 (18.95) & $1(6.25)$ \\
\hline \multicolumn{3}{|l|}{ Baseline IS regimen } \\
\hline TAC & 71 (74.74) & $15(93.75)$ \\
\hline MMF & $43(45.26)$ & $4(25)$ \\
\hline Prednisone & $21(22.11)$ & $4(25)$ \\
\hline EVE & $7(7.37)$ & $0(0)$ \\
\hline Methylprednisolone & $9(9.47)$ & $1(6.25)$ \\
\hline$A Z A$ & $6(6.32)$ & $0(0)$ \\
\hline MPA & $3(3.16)$ & $0(0)$ \\
\hline Cyclosporine & 1 (1.05) & $0(0)$ \\
\hline Corticosteroid NS & $1(1.05)$ & $3(18.75)$ \\
\hline Methotrexate & $1(1.05)$ & $0(0)$ \\
\hline SIR & $0(0)$ & $2(12.5)$ \\
\hline NS & $7(7.37)$ & $0(0)$ \\
\hline
\end{tabular}

*The bold line separates the adult data (above the line) from the pediatric data (below the line). ALT: alanine aminotransferase; AST: aspartate aminotransferase; AZA: azathioprine; CRP: C-reactive protein; EVE: Everolimus; GGT: gamma-glutamyltransferase; IS: immunosuppression; MMF: mycophenolate mofetil; MPA: mycophenolic acid; NS: not specified; SIR: sirolimus; TAC: tacrolimus; UK: United Kingdom; USA: United States of America; WBC: white blood cells. 
A significant correlation between age and outcome was identified $\left(r_{s}=-0.235, p=0.022\right)$, meaning that older age is associated with a worse outcome (death). Moreover, age was also associated with graft rejection $\left(r_{s}=-0.409\right.$, $p<0.001)$ and with neurological complications $\left(r_{s}=0.315\right.$, $p=0.035)$, meaning that older age is associated with no graft rejection and with neurological complications.

No statistical association was found between time after LT and the outcome $\left(r_{s}=0.037, p=0.726\right)$. However, time after LT had significant correlations with graft rejection $\left(r_{s}=-0.362, p=0.016\right)$ and presence of other complications during treatment $\left(r_{s}=-0.360, p=0.016\right)$ (except for ARDS, AKI, pneumonia, shock and neurological complications).

Regarding comorbidities, diabetes was associated with a worse outcome $(B=-2.033, p=0.043,95 \% \mathrm{Cl}=-54.065$; $-0.065)$ and obesity/overweight was associated with a better outcome $(B=2.528, p<0.001,95 \% \mathrm{Cl}=0.000$; $89,467)$. No significant statistical associations were found between all other comorbidities (AHT, CKD, CVD, respiratory disease, gastrointestinal disease and HIV), and between complications during treatment.

MMF as a baseline IS regimen was associated with complications during treatment $(60 \%$ of the cases with complications were on MMF, and $100 \%$ of the cases with no complications were not on MMF) and with shock ( $\mathrm{X}^{2}{ }_{[1]}$ $=5.200, p=0.023)$. An association between AZA and ARDS was found $(X 2[1]=5.645, p=0.018)$. Furthermore, statistical significance was found between cyclosporine and $\mathrm{AKI}\left(\mathrm{X}^{2}{ }_{[1]}=6.979, \mathrm{p}<0.001\right)$, methylprednisolone and graft rejection $\left(X^{2}{ }^{21]}=10.900, p<0.001\right)$, and between an unspecified corticoid and graft rejection $\left(\mathrm{X}^{2}{ }^{[1]}=8.980\right.$, $p<0.001)$. No statistical significance was found between any baseline IS regimen and the outcome. The presence of initial symptoms was associated with complications during treatment when compared to asymptomatic patients $\left(X^{2}[1]=9.124, p<0.001\right)$. In fact, some symptoms were associated with specific complications, mainly fever with neurological complications $\left(X^{2}[1]=9.124, p<0.001\right)$ and fatigue to $\mathrm{AKI}\left(\mathrm{X}^{2}{ }^{[1]}=6.723, \mathrm{p}=0.01\right)$. SOB, cough and headache were also associated with complications in general $\left(X^{2}{ }^{11]}=8.862, p<0.001 ; X^{2}{ }_{[1]}=10.820, p<0.001\right.$; and $\mathrm{X}^{2}{ }^{[1]}=4.864, \mathrm{p}=0.027$; respectively). Regarding the outcome, patients with cough and diarrhea were associated with worse outcome $\left(X^{2}{ }^{[1]}=5.309, p=0.021\right.$; and $X^{2}{ }^{[1]}=8.594, p=0.003$ ).

Statistical significance was also found between some of the laboratory results and the outcome. A higher level of WBC was associated with a worse outcome $(B=-0.188$, $p=0.015,95 \% \mathrm{Cl}=0.713 ; 0,964)$ and higher levels of AST were also associated with worse final results $(B=-$ $0.016, p=0.01,95 \% \mathrm{Cl}=0.971 ; 0,996)$. Regarding the significance between main laboratory analyzes and complications, lymphopenia was associated with a severe evolution $(B=-1.953, p=0.036,95 \% \mathrm{Cl}=0.023$;
$0,884)$ as well as higher levels of $C R P(B=0.173, p=0.048$, $95 \% \mathrm{Cl}=1.001 ; 1.412$ ).

None of the drugs used in other pharmacological therapy (HCQ, tocilizumab, lopinavir/ritonavir, remdesivir, umifenovir, oseltamivir, IFN-a, IFN-b, azithromycin, other antibiotics and fresh convalescence plasma) were associated with the outcome. Nonetheless, the use of other pharmacological therapy was associated with complications in general $\left(X^{2}{ }^{[1]}=4.648, p=0.031\right)$. Azithromycin demonstrated a dependency relationship with pneumonia $\left(X^{2}{ }^{[1]}=5.692, p=0.017\right)$.

Support ventilation had also shown a statistical dependence on complications during treatment $\left(X^{2}{ }_{[1]}=\right.$ $21.860, p<0.01)$. In fact, $93.5 \%$ of patients with support ventilation developed at least one complication during hospital stay, while only $6.5 \%$ of the cases with complications were not on support ventilation. Moreover, ventilation and outcome had presented statistical dependence $\left(X^{2}{ }_{[1]}=13.935, p<0.001\right)$, with $100 \%$ of patients with no ventilation alive. Mechanical ventilation was associated with poor outcome $\left(\mathrm{X}^{2}{ }_{[1]}=\right.$ 16.593, $p<0.001)$ and ECMO with ARDS $\left(X^{2}{ }_{[1]}=4.971\right.$, $p=0.026)$ and $A K I\left(X^{2}[1]=4.971, p=0.026\right)$.

\section{Pediatric population}

Regarding the pediatric population, seven $(43.75 \%)$ were male, and nine (56.25\%) were female. The mean age of patients was 1.28 years ( $\sigma=1.49$ years), ranging from 0.5 to 4.58 years. In $10(62.5 \%)$ cases the age of the patient have not been specified. Regarding the race/ethnicity of the patient, seven (43.75\%) were Hispanic, four (25\%) white, and five (31.25\%) have not been specified. The mean time from liver transplantation to SARS-CoV-2 infection diagnosis was 3.50 years $(\sigma=4.80)$, ranging from 0.01 to 18.01 years.

The most frequent cause for liver transplantation was biliary atresia ( $n=5 ; 31.25 \%$ ), followed by cryptogenic cirrhosis $(n=1 ; 6.25 \%)$, cholestatic liver disease $(n=1$; $6.25 \%$ ), and hepatic adenomatosis ( $n=1 ; 6.25 \%)$. In 8 $(50 \%)$ cases, the cause of liver transplantation has not been specified. Obesity/overweight was the most common comorbidity present in three $(18.75 \%)$ cases, followed by $\mathrm{AHT}$, diabetes mellitus, asthma and hepatoblastoma in two (12.5\%) cases, and CKD, hypothyroidism and Ellis-van Creveld syndrome in one $(6.25 \%)$ case. In five $(31.25 \%)$ cases there were no comorbidities, and in one $(6.25 \%)$ case it was not specified. The most used baseline immunosuppressive regimen in monotherapy or associated with another immunosuppressant was TAC (15 [93.75\%] cases) followed by MMF (four [25\%] cases), prednisone (four [25\%] cases), corticosteroids (three [18.75\%] cases), SIR (two [12.5\%] cases), and methylprednisolone (one [6.25\%] case). Table 2 presents all pediatric demographic data. 
Fever was identified as a primary symptom in eight $(50 \%)$ cases, cough in six $(37.5 \%)$ cases, SOB in four $(25 \%)$ cases, diarrhea in three $(18.75 \%)$ cases, chest pain in two $(12.5 \%)$ patients, and sore throat, rhinorrhea, headache, respiratory deterioration and nasal congestion in one $(6.25 \%)$ patient. The time between the unset of COVID-19 symptoms to hospital admission ranged between 0 and 3 days (mean of 0.5 days; $\sigma=$ 1.12). In 10 (62.5\%) cases, it was not specified.

The only data regarding $\mathrm{SpO} 2$ was found in only two $(12.5 \%)$ cases presenting $96 \%$ and $99 \%$ of peripheral oxygen saturation (mean of $97.5 \% ; \sigma=2.12 \%$ ). The values of hemoglobin were within the normal range in nine $(56.25 \%)$ cases, lower in one $(6.25 \%)$ case, and not specified in six $(37.5 \%)$ cases. WBC count was normal in seven $(43.75 \%)$ cases, higher in one $(6.25 \%)$ patient (value of $18 \times 10^{9}$ cells/L), lower in three $(18.75 \%$ ) cases, and not specified in five $(31.25 \%)$ cases. Absolute lymphocyte count was normal in 11 (68.75\%) cases, and not specified in five $(31.25 \%)$ cases. The platelet count was normal in $10(62.5 \%)$ patients, although not specified in the other six $(37.5 \%)$ cases. ALT and AST were specified in only three patients, presenting values of 980,3 and $1253 \mathrm{U} / \mathrm{L}$ (mean of $743.33 \mathrm{U} / \mathrm{L} ; \sigma=657,21$ $\mathrm{U} / \mathrm{L}$ ), and 908,4 and $908 \mathrm{U} / \mathrm{L}$ (mean of $606.67 \mathrm{U} / \mathrm{L} ; \sigma$ $=521,93 \mathrm{U} / \mathrm{L}$ ), respectively. In the remaining patients, ALT and AST values were not specified. GGT was also specified in the same three patients, with values of 656 , 5 and $473 \mathrm{U} / \mathrm{L}$ (mean of $378 \mathrm{U} / \mathrm{L} ; \sigma=335,74 \mathrm{U} / \mathrm{L}$ ). In 15 $(93.75 \%)$ patients, tBRB have not been specified. The only tBRB value presented was $3.6 \mathrm{md} / \mathrm{dL}$. CRP was high in three $(18.75 \%)$ patients, normal in four $(25 \%)$ cases and not specified in nine $(56.25 \%)$ other cases (mean of $2.48 \mathrm{mg} / \mathrm{dL} ; \sigma=2.16 \mathrm{mg} / \mathrm{dL}$ ). IL-6 have not been specified in any of the patients. Finally, ferritin was normal in five $(31.25 \%)$ cases, while not specified in the remaining $11(68.75 \%)$ cases.

A total of five $(31.75 \%)$ patients were admitted to the ICU. Regarding the IS management, it was withdrawn in one $(6.25 \%)$ case, the dose was lowered in three $(18.75 \%)$ cases, a new immunosuppressant was added in one $(6.25 \%)$ case, not changed in nine $(56.25 \%)$ cases and not specified in three $(18.75 \%)$ cases. Azithromycin was added in four $(25 \%)$ cases, followed by $\mathrm{HCQ}(\mathrm{n}=3 ; 18.75 \%)$, other antibiotics ( $\mathrm{n}$ $=2 ; 12.5 \%)$, and immunoglobulin $(n=1 ; 6.25 \%)$. In six $(37.5 \%)$ cases no additional pharmacological therapy was prescribed, and in three $(18.75 \%)$ cases it have not been specified.

ECMO was necessary in three $(18.75 \%)$ patients, CPAP in two (12.5\%) patients, and in $11(68.75 \%)$ cases support ventilation was not necessary. The most frequent complication during admission was ARDS ( $n=3 ; 18.75 \%)$, followed by acute liver rejection ( $n=2 ; 12.5 \%$ ), and hepatitis ( $n=1 ; 6.25 \%)$. No complications were found in 11 $(68.75 \%)$ cases. In one $(6.25 \%)$ patient the complications during hospital stay have not been specified.

By the end, $13(81.25 \%)$ patients were alive and three $(18.75 \%)$ had passed away. The mean time for follow-up/ hospital stay was 14.83 days ( $\sigma=11.07$ days), ranging from 4 to 36 days. In 10 (62.5\%) patients, this information have not been specified. Table 1 presents a summary of each clinical case.

\section{DISCUSSION}

In our study, we tried to identify prognosis factors for disease severity and worse outcome. As expected, older age was a poor outcome factor. This may be explained by physiological modifications, typical of elderly people that cause frailty and susceptibility to more diseases. Another finding was that the severity and outcome were not affected by the post-LT time. This hypothesis is due to, as in most cases, patients with a shorter post-LT time are on higher doses of immunosuppressant, this leads to a more severe immunosuppression, and consequently, an increased disease severity. In a study conducted by Malekhosseini et al. in Iran, ${ }^{60}$ he reported a total of 85 cases of COVID-19, being 66 from LT (77.65\%), 16 from kidney transplantation (18.82\%), two from kidneypancreas transplantation $(2.35 \%)$ and one from liverkidney transplantation (1.18\%). The average age of their patients was 46.4 years and the main comorbidities were diabetes $(25.9 \%)$ and AHT (18.8\%). Overall, 17 patients died, and $21.9 \%$ were LT recipients. Although including some transplants other than liver, their results were very similar to ours (medium age of 58.73 years and overall death of $20 \%$ ). However, the authors referred to an association between the post-LT time and the outcome: they reported that of five patients who had LT within one month, four died. Another point of view is presented by Belli et al. ${ }^{61}$ and by Bhoori et al. ${ }^{16}$ in their correspondence, and by Merli et al. ${ }^{62}$ in their letter to the editor. All concluded that mortality could be worse in patients with longer LT time. Both these associations were not found in our report $(p=0.726)$, as well as in other similar case series. ${ }^{9}$

It is unanimous that the presence of comorbidities is associated with a poor outcome and/or disease severity. The most frequent comorbidities in our report were AHT and diabetes. This fact is similar in many other case series. ${ }^{63}$ In our study, the only comorbidity associated with a worse prognosis was diabetes. In fact, in our report, the probability of a diabetic patient to die after 
being infected with SARS-CoV-2 is $7 .{ }^{63}$ higher than a non-diabetic patient. Diabetes is a multifactorial disease with long-term metabolic and vascular abnormalities and a higher susceptibility to infectious diseases. Insulin resistance and hyperglycemia result in a promotion of pro-inflammatory cytokines, oxidative stress and stimulating the production of mediators of tissue inflammation. ${ }^{64}$ This pro-inflammatory status may be the underlying cause for this relation between diabetes and COVID-19.

An interesting finding was that obesity/overweight was a protective factor for worse outcome. Obese/ Overweight patients with COVID-19 have 12.54 more chance of being alive than patients with normal/ low weight. This may be evidence for the "obesity survival paradox" (a higher severity of disease, but a lower mortality rate in obese patients), which is present in other diseases, such as pneumonia. ${ }^{65}$ Although this relation is still very controversial, some pathophysiological mechanisms can partially explain this phenomenon: a higher level of serum cholesterol may bind endotoxin and the excess of energy stored in adipose tissues, and probably others that are still unidentified. ${ }^{65} \mathrm{~A}$ larger study focused on overweight and obese patients with COVID-19 should provide some help in understanding this relationship. CVD was not associated with a poor outcome. This finding was similar to an international European liver transplant recipient cohort by Becchetti et al. ${ }^{12}$ who included 57 $\mathrm{LT}$ recipients infected with SARS-CoV-2.

Another interesting finding was that MMF was associated with more complications and with shock. MMF is an antimetabolite drug that disrupts the making of RNA and DNA, thus preventing the replication of $B$ and $\mathrm{T}$ lymphocytes. ${ }^{18}$ When infected by SARS-CoV-2, the levels of these lymphocytes in the patient, plus NK cells, face a significant decrease in the total number, and consequently, a dysregulation of immune response, thus being more prone to other infections and septic shock. In fact, this evidence is considered by some liver societies. British Transplant Society advocates that any LT recipient with a positive COVID-19 test should stop MMF. ${ }^{66}$ The same position is taken by the Spanish Society of Liver Transplantation, adding that if MMF regimen is in monotherapy, a conversion to CNIs, mTOR inhibitors or steroids should be considered, ${ }^{67}$ and by the American Association for the Study of Liver Diseases, which advises that all LT recipients should suspend or reduce the dose of MMF.68

It is evidenced in the literature that CNIs, especially cyclosporine, can cause AKI by strongly constricting the afferent renal arteriole, concomitantly, activating the renin-angiotensin-aldosterone system leading to an impaired renal function. ${ }^{69}$ This may be the reason that in our work we found a positive association between cyclosporine and $\mathrm{AKI}$. In this case, it is recommended to decrease the cyclosporine dose or to replace it by steroids, to avoid graft rejection. A study led by Lee et al. from Recanati/Miller Transplantation Institute ${ }^{28}$ with 38 LT recipients infected with SARS-CoV-2 reached the same association between CNIs and AKI.

Another conclusion that can be drawn is regarding the laboratory data. In our report, we found out that higher levels of WBCs and AST can be associated independently to a worse outcome. In a multicenter observational cohort study of 112 LT recipients with COVID-19, Rabiee and Sadowski et al. ${ }^{63}$ revealed that liver injury is an independent factor for increased mortality. They showed that patients with a peak of liver enzymes during SARS-CoV-2 infection had a worse outcome. Although their focus was on ALT, as it is more specific to the liver than AST, they conclude that all liver enzymes should be closely monitored during hospital stay as they can predict a poor ending.

A pro-inflammatory state can also be associated with increased mortality and with a more severe course of the disease. This is supported by a positive association by increasing WBCs levels and mortality, and by higher levels of CRP and a higher complication rate. Moreover, lymphopenia was also associated to an increased disease severity. The infection by SARS-CoV-2 is known to create lymphopenia, and in a severe form of the disease, it is characterized by an excessive pro-inflammatory production mainly by IL-2, IL-7, IL-6 and TNF-a.18 This phenomenon associated with an incompetent immune system, is responsible by the severity and mortality of this disease. Similar to our study, Iymphopenia was present in many other series. ${ }^{28,60}$

None of the specific pharmacological therapies were associated with the outcome in our study. Most of patients in our series had done HCQ or lopinavir/ritonavir during hospital stay, as a targeted therapy for COVID-19. Although guidelines now prove the ineffectiveness of these drugs, most of our cases were from the initial days of the pandemic, and these two drugs were the first approved for SARS-CoV-2 infection. In fact, because of the interactions with the most used immunosuppressant, there is an increased risk for graft failure if the IS is not strictly regulated. Regarding the association between azithromycin and pneumonia, we interpret it as being used as a therapeutic, as this antibiotic is used in most cases, as a first-line therapy.

In our study, $16.84 \%$ of the adult population was admitted to the ICU. In the study referred before, Malekhosseini et al. ${ }^{60}$ reported a slightly higher ICU admission with $33.9 \%$ 
of cases. The authors also described $20 \%$ mortality, being the same in our study. In another series, Belli et al. ${ }^{61}$ presented an ICU admission rate of $15 \%$ in his 103 LT recipients. He also reported that $66 \%$ of patients required support ventilation (vs $44.21 \%$ in our work) and, of those, $15 \%$ needed invasive ventilation (vs $12.63 \%$ ). His overall mortally rate for LT recipients was $16 \%$ (vs $20 \%$ ). Finally, in a series by Coll et al., ${ }^{70}$ he reported a death rate of $22 \%$ between his 76 LT recipients. According to the WHO, the mortality rate of COVID-19 in the general population is $2.22 \% .^{2}$ When comparing the overall mortality with the one from our study $(2.22 \%$ vs $20 \%$, respectively) we can conclude that LT recipients have a death rate almost 10 times higher. This may be evidence that LT recipients with COVID-19 are at higher risk of dying.

We also included some pediatric patients in our study. However, because of the low number of cases and the missing data, no statistical conclusions were made. D'Antiga et al. ${ }^{17}$ presented a study with 700 children on which only three were infected with SARS-CoV-2, and none died, concluded that the pediatric population was not at higher risk for the disease severity and mortality. Another series from Italy led by Doná ${ }^{71}$ presented an European survey on transplantation centers that reported three LT recipients with COVID-19. All patients had mild symptoms and none died. In our study, the mortality rate was $18.75 \%$. This overall mortality in the pediatric population must be taken carefully, as only 16 single cases were analyzed, and mortally was only present in one article. In this work, Imam et al. ${ }^{56}$ presented three liver pediatric recipients with COVID-19. The first case was a 10-month-old male that, on the eighth post-operatory day developed fever and SOB that causes the need for ECMO, and the patient eventually died. The second case was also a 10-month male that also developed fever and needed ECMO. In this case, a high level of liver enzymes and neutropenia was found. The final case was a 5-month male that needed ECMO for worsen respiratory function on day three, and died on that same day.

COVID-19 is also known to cause myocardial lesions like atrial fibrillation, systolic/diastolic abnormalities and ischemic or non-ischemic cardiomyopathy, resulting from direct viral invasion, thromboembolism, STEMI and/ or pro-inflammatory state. In fact, measuring the levels of troponins and BNP/Pro-BNP could help to identify these patients. ${ }^{72}$ Myocardial lesion was absent in all of the cases analyzed for this research.

Another known fact of SARS-CoV-2 infection is that it may lead to long-term sequelae. The main longterm symptoms can be respiratory (cough, SOB), cardiovascular (palpitations, chest pain, thrombotic events), neurological (cognitive impairment, headache, peripheral neuropathic symptoms, delirium), general (fatigue, fever, pain), and others. ${ }^{73}$ During our research period, no study on long-term complications in LT recipients was found. In our study, the follow-up ranges between 1 day and 75 days (mean of 21.7 days). Therefore, when accessing the main long-term complications by a close follow-up of the surviving patients, it could be a very interesting and important investigation.

Our study had some limitations. The main limitation was regarding the missing data. This may have contributed to an absence of association between some of the variables, for example, only 9 of 95 adult cases have platelet count and 48 of 95 have CRP serum value. In order to reduce the missing data, it is important to publish the complete clinical information, IS management, laboratory finds, as well as specific COVID-19 therapy, if available. A possible bias is related to a sample with only symptomatic patients tested, or at risk contact with an infected patient during hospital stay. This fact may justify the overall increased mortality in LT recipients because, when comparing to the overall population, no mass screenings were conducted in this specific population, resulting in an under diagnosis of asymptomatic LT recipients.

\section{CONCLUSION}

Older age and diabetes were independently associated with the worse outcome, as well as a higher level of WBC and the need for support ventilation. On the another hand, higher levels of CRP and lower levels of lymphocytes were associated with a more severe course of the disease. Obesity or overweight, interestingly, was associated with a lower death rate, proving the evidence of an "obesity survival paradox". An important association between MMF as a baseline IS regimen and shock during hospital stay was found. No association between post-LT time to COVID-19, specific antiviral therapy and outcome were found. Overall, liver transplantation recipients may be at higher risk of dying with the infection of SARS-CoV-2. 


\section{RESUMO}

Introdução: Devido à pandemia COVID-19 e ao facto dos recetores de transplante hepático serem uma população imunodeprimida, iniciou-se discussão sobre o risco acrescido nesta população: existe controvérsia na identificação de fatores de pior prognóstico, mas também no manuseamento da imunossupressão de manutenção e terapia específica para a COVID-19. Objetivo: O presente trabalho pretende analisar publicações (casos e séries) sobre fatores demográficos de risco, imunossupressão de base e manuseamento, terapia específica para COVID-19 e resultados em transplantados hepáticos. Material e Métodos: Foi realizada pesquisa nas bases de dados MEDLINE e PubMed. Foram identificados 127 artigos e 55 incluídos na análise quantitativa final. Utilizaram-se os testes chi-quadrado, correlação de spearman e regressão logística para análise estatística. As análises descritivas são apresentadas com número e percentagem, juntamente com média e intervalo de alcance. Resultado: Foram analisados 111 casos clínicos (95 adultos e 16 pediátricos). No que diz respeito a adultos, $69.47 \%$ eram homens, e a média da idade foi de 58.73 anos. As co-morbilidades mais comuns foram: obesidade/excesso de peso (35.79\%), hipertensão arterial (33.68\%) e diabetes (27.37\%). Os imunossupressores mais utilizados foram o tacrolimus (TAC) $(74.74 \%)$ e micofenolato mofetil (MMF) (45.26\%). Quarenta e um doentes (43.16\%) apresentaram complicações durante o tratamento e 12 (12.63\%) necessitaram de ventilação invasiva. A taxa de mortalidade referente à população adulta foi de $20 \%$. Quanto à população pediátrica, $43.75 \%$ eram rapazes e a idade média foi de 1.28 anos. O imunossupressor mais utilizado foi o TAC (93.75\%). Apenas quatro doentes (25\%) apresentaram complicações e três (18.75\%) precisaram de ventilação invasiva. A mortalidade da população pediátrica foi de $18.75 \%$. Conclusão. Idade avançada, diabetes, neutrofilia e o uso de ventilação foram associados a pior prognóstico. Elevados níveis de proteína $\mathrm{C}$ reativa e linfopenia foram associados a severidade da doença. O uso de MMF foi associado a choque sético durante o internamento. A mortalidade nos transplantados hepáticos infetados com COVID-19 foi superior à da população em geral.

Descritores: Transplantação Hepática; COVID-19; Complicações pós-operatórias.

\section{LIST OF ABBREVIATIONS}

AHT - arterial hypertension; AI - autoimmune; AKI - acute kidney injury; ALF - acute liver failure; ALT - alanine aminotransferase; ARDS - acute respiratory distress syndrome; AST - aspartate aminotransferase; AZA - azathioprine; BiPAP - bilevel positive airway pressure; BNP - brain natriuretic peptide; CKD - chronic kidney disease; CNI calcineurin inhibitor; COPD - chronic obstructive pulmonary disease; COVID-19 - coronavirus infectious disease 2019; CPAP - continuous positive airway pressure; CRP - C-reactive protein; CVD - cardiovascular disease; DM diabetes mellitus; EBV - Epstein-Barr virus; CMO - extracorporeal membrane oxygenation; EVE - everolimus; GGT - gamma-glutamyltransferase; HBV - hepatitis B virus; HCC - hepatocellular carcinoma; HCQ - hydroxychloroquine; HCV - hepatitis C virus; HIV - human immunodeficiency virus; ICU - intensive care unit; INF-a - interferon alpha; IFN-b - interferon beta; IL - interleukin; IS - immunosuppression; LMWH - low molecular weight heparin; LT - liver transplantation; MeSH - medical subject headings; MMF - mycophenolate mofetil; MPA - mycophenolic acid; mTOR - mammalian target of rapamycin; NASH - nonalcoholic steatohepatitis; NS - not specified; PCR - polymerase chain reaction; PICO - population, intervention, control/comparison, outcome; PHEIC - public health emergency of

international concern; PRISMA - preferred reporting items for systematic reviews and meta-analyses; PSC - primary sclerosing cholangiopathy; ReTx - re-transplantation; rhG-CSF - recombinant human granulocyte colony-stimulating factor; SARS-CoV-2 - severe acute respiratory syndrome coronavirus 2; SIR - sirolimus; SOB - shortness of breath; SOFA - Sequential Organ Failure Assessment; SpO2 - peripheral oxygen saturation; STEMI - ST elevated myocardial infarction; TAC - tacrolimus; tBRB - total bilirubin; UK - United Kingdom; USA - United States of America; WBC white blood cells; WHO - World Health Organization; 


\section{REFERÊNCIAS}

1. World Health Organization. Timeline: WHO's COVID-19 response 2020 [Available from: https://www.who.int/ emergencies/diseases/novel-coronavirus-2019/interactivetimeline\#!

2. World Health Organization. Coronavirus disease (COVID-19) pandemic: Numbers at a glance. 2020 [Available from: https://www.who.int/emergencies/diseases/novelcoronavirus-2019.

3. Henriques G, Rato Nunes R, Henriques S. Portugal confirma dois casos de Covid-19 com ligações a Itália e Espanha Diário de Notícias: Diário de Notícias; 2020 [Available from: https://www.dn.pt/pais/confirmados-dois-primeiros-casosde-coronavirus-em-portugal-11805920.html.

4. Diário da República. Decreto do Presidente da República $n$. 14-A/2020. 2020. p. 13-(2-4).

5. Ministério da Saúde. Ponto de Situação Atual em Portugal 2020 [Available from: https://covid19.min-saude.pt/.

6. Imam A, Abukhalaf SA, Merhav H, Abu-Gazala S, CohenArazi O, Pikarsky AJ, et al. Prognosis and Treatment of Liver Transplant Recipients in the COVID-19 Era: A Literature Review. Ann Transplant. 2020;25:e926196.

7. Zhou F, Yu T, Du R, Fan G, Liu Y, Liu Z, et al. Clinical course and risk factors for mortality of adult inpatients with COVID-19 in Wuhan, China: a retrospective cohort study. Lancet. 2020;395(10229):1054-62.

8. Nacif LS, Zanini LY, Waisberg DR, Pinheiro RS, Galvão F, Andraus W, et al. COVID-19 in solid organ transplantation patients: A systematic review. Clinics (Sao Paulo). 2020;75:e1983.

9. Webb GJ, Marjot T, Cook JA, Aloman C, Armstrong MJ, Brenner EJ, et al. Outcomes following SARS-CoV-2 infection in liver transplant recipients: an international registry study. Lancet Gastroenterol Hepatol. 2020;5(11):1008-16.

10. Guan WJ, Liang WH, Zhao Y, Liang HR, Chen ZS, Li YM, et al. Comorbidity and its impact on 1590 patients with COVID-19 in China: a nationwide analysis. Eur Respir J. 2020;55(5).

11. Zhang J, Yu M, Tong S, Liu LY, Tang LV. Predictive factors for disease progression in hospitalized patients with coronavirus disease 2019 in Wuhan, China. J Clin Virol. 2020;127:104392.

12. Becchetti C, Zambelli MF, Pasulo L, Donato MF, Invernizzi F, Detry O, et al. COVID-19 in an international European liver transplant recipient cohort. Gut. 2020;69(10):1832-40.

13. Tan L, Wang Q, Zhang D, Ding J, Huang Q, Tang YQ, et al. Correction: Lymphopenia predicts disease severity of COVID-19: a descriptive and predictive study. Signal Transduct Target Ther. 2020;5:61.

14. Jeannet R, Daix T, Formento R, Feuillard J, François B. Severe COVID-19 is associated with deep and sustained multifaceted cellular immunosuppression. Intensive Care Med. 2020;46(9):1769-71.

15. Kumar D, Manuel O, Natori Y, Egawa H, Grossi P, Han SH, et al. COVID-19: A global transplant perspective on successfully navigating a pandemic. Am J Transplant. 2020;20(7):1773-9.
16. Bhoori S, Rossi RE, Citterio D, Mazzaferro V. COVID-19 in long-term liver transplant patients: preliminary experience from an Italian transplant centre in Lombardy. Lancet Gastroenterol Hepatol. 2020;5(6):532-3.

17. D'Antiga L. Coronaviruses and Immunosuppressed Patients: The Facts During the Third Epidemic. Liver Transpl. 2020;26(6):832-4.

18. Parente A, Manzia T, Angelico R, Tirotta F, Muiesan $\mathrm{P}$, Tisone $\mathrm{G}$, et al. COVID-19, liver transplant, and immunosuppression: Allies or foes? Transpl Infect Dis. 2020;00:e13417.

19. article CttC. C4 article: Implications of COVID-19 in transplantation. Am J Transplant. 2020.

20. Boettler T, Newsome P, Mondelli M, Maticic M, Cordero $E$, Cornberg $M$, et al. Care of patients with liver disease during the COVID-19 pandemic: EASL-ESCMID position paper. JHEP Rep 2020;2(3):100113.

21. Moher D, Liberati A, Tetzlaff J, Altman DG, Group P. Preferred reporting items for systematic reviews and meta-analyses: the PRISMA statement. Int J Surg. 2010;8(5):336-41.

22. Fernández-Ruiz M, Andrés A, Loinaz C, Delgado JF, López-Medrano F, San Juan R, et al. COVID-19 in solid organ transplant recipients: A single-center case series from Spain. Am J Transplant. 2020;20(7):184958.

23. Zibiao Z. Clinical characteristics and immunosuppressant management of coronavirus disease 2019 in solid organ transplant recipients In: Quyan Z, Haoyang X, Aiping W, Wenjin L, Wei Z, Lihua Z, et al., editors. Am J Transplant2020. p. 1916-21.

24. Qin J, Wang H, Qin X, Zhang P, Zhu L, Cai J, et al. Perioperative Presentation of COVID-19 Disease in a Liver Transplant Recipient. Hepatology. 2020.

25. Huang JF, Zheng KI, George J, Gao HN, Wei RN, Yan $H D$, et al. Fatal outcome in a liver transplant recipient with COVID-19. Am J Transplant. 2020;20(7):1907-10.

26. Liu B, Wang Y, Zhao Y, Shi H, Zeng F, Chen Z. Successful treatment of severe COVID-19 pneumonia in a liver transplant recipient. Am J Transplant. 2020;20(7):1891-5.

27. Hammami MB, Garibaldi B, Shah P, Liu G, Jain T, Chen $\mathrm{PH}$, et al. Clinical course of COVID-19 in a liver transplant recipient on hemodialysis and response to tocilizumab therapy: A case report. Am J Transplant. 2020;20(8):2254-9.

28. Lee BT, Perumalswami PV, Im GY, Florman S, Schiano TD, Group CS. COVID-19 in Liver Transplant Recipients: An Initial Experience From the US Epicenter. Gastroenterology. 2020;159(3):1176-8.e2.

29. Hoek RAS, Manintveld OC, Betjes MGH, Hellemons ME, Seghers L, Van Kampen JAA, et al. COVID-19 in solid organ transplant recipients: a single-center experience. Transpl Int. 2020;33(9):1099-105. 
30. Kates OS, Fisher CE, Stankiewicz-Karita HC, Shepherd AK, Church EC, Kapnadak SG, et al. Earliest cases of coronavirus disease 2019 (COVID-19) identified in solid organ transplant recipients in the United States. Am J Transplant. 2020;20(7):1885-90.

31. Gao F, Zheng KI, Gu JY, George J, Zheng MH. COVID-19 and liver transplantation: Lessons learned from three reported cases. Transpl Infect Dis. 2020;22(4):e13335.

32. Verma A, Khorsandi S, Dolcet A, Prachalias A, Suddle A, Heaton $\mathrm{N}$, et al. Low prevalence and disease severity of COVID-19 in post-liver transplant recipients - A single centre experience. Liver Int. 2020;40(8):1972-6.

33. Fung M, Chiu C, DeVoe C, Doernberg S, Schwartz B, Langelier $C$, et al. Clinical outcomes and serologic response in solid organ transplant recipients with COVID-19: A case series from the United States. Am J Transplant. 2020;20(11):3225-33.

34. Müller H, Kniepeiss D, Stauber R, Schrem H, Rauter M, Krause R, et al. Recovery from COVID-19 following hepatitis $C$, human immunodeficiency virus infection, and liver transplantation. Am J Transplant. 2020;20(11):3255-6.

35. Patrono D, Lupo F, Canta F, Mazza E, Mirabella S, Corcione S, et al. Outcome of COVID-19 in liver transplant recipients: A preliminary report from Northwestern Italy. Transpl Infect Dis. 2020;22(5):e13353.

36. John Hann A, Lembach H, McKay SC, Perrin M, Isaac J, Oo $\mathrm{YH}$, et al. Controversies regarding shielding and susceptibility to COVID-19 disease in liver transplant recipients in the United Kingdom. Transpl Infect Dis. 2020;22(5):e13352.

37. Donato MF, Invernizzi F, Lampertico P, Rossi G. Health Status of Patients Who Underwent Liver Transplantation During the Coronavirus Outbreak at a Large Center in Milan, Italy. Clin Gastroenterol Hepatol. 2020;18(9):2131-3.e1.

38. Mehta SA, Rana MM, Motter JD, Small CB, Pereira MR, Stosor V, et al. Incidence and Outcomes of COVID-19 in Kidney and Liver Transplant Recipients With HIV: Report From the National HOPE in Action Consortium. Transplantation. 2021;105(1):216-24.

39. Prieto M, Gastaca M, Ruiz P, Ventoso A, Palomares I, Rodríguez-Álvarez R, et al. A case of COVID-19 immediately after liver transplantation: Not only bad news. Ann Hepatobiliary Pancreat Surg. 2020;24(3):314-8.

40. Jamir I, Lohia P, Pande RK, Setia R, Singhal AK, Chaudhary A. Convalescent plasma therapy and remdesivir duo successfully salvaged an early liver transplant recipient with severe COVID-19 pneumonia. Ann Hepatobiliary Pancreat Surg. 2020;24(4):526-32.

41. Eslami P, Moradi M, Dooghaie Moghadam A, Pirsalehi A, Abdul Lateef S, Hadaegh A, et al. Lethal outcome of Covid-19 pneumonia in a new liver recipient with neurological manifestation. Gastroenterol Hepatol Bed Bench. 2020;13(4):405-9.

42. García-Juárez I, Campos-Murguía A, Tovar-Méndez V, Gabutti A, Ruiz I. Unexpected better outcome in a liver transplant recipient with COVID-19: a beneficial effect of tacrolimus? Rev Gastroenterol Mex. 2020;85(4):437-42.
43. Hatami B, Ketabi Moghadam P, Zali M. Presentation of COVID-19 in a liver transplant recipient. Gastroenterol Hepatol Bed Bench. 2020;13(4):396-9.

44. Bösch F, Börner N, Kemmner S, Lampert C, Jacob S, Koliogiannis $D$, et al. Attenuated early inflammatory response in solid organ recipients with COVID-19. Clin Transplant. 2020;34(10):e14027.

45. Antony SJ, Singh J, de Jesus M, Lance J. Early use of tocilizumab in respiratory failure associated with acute COVID -19 pneumonia in recipients with solid organ transplantation. IDCases. 2020;21:e00888.

46. Loinaz C, Marcacuzco A, Fernández-Ruiz M, Caso O, Cambra F, San Juan R, et al. Varied clinical presentation and outcome of SARS-CoV-2 infection in liver transplant recipients: Initial experience at a single center in Madrid, Spain. Transpl Infect Dis. 2020;22(5):e13372.

47. Modi AR, Koval CE, Taege AJ, Modaresi Esfeh J, Eghtesad B, Narayanan Menon KV, et al. Coronavirus disease 2019 in an orthotopic liver transplant recipient living with human immunodeficiency virus. Transpl Infect Dis. 2020;22(5):e13351.

48. Felldin M, Søfteland JM, Magnusson J, Ekberg J, Karason K, Schult A, et al. Initial Report From a Swedish Highvolume Transplant Center After the First Wave of the COVID-19 Pandemic. Transplantation. 2021;105(1):108-14.

49. Kolonko A, Dudzicz S, Wiecek A, Król R. COVID-19 infection in solid organ transplant recipients: A singlecenter experience with patients immediately after transplantation. Transpl Infect Dis. 2020:e13381.

50. Sessa A, Mazzola A, Lim C, Atif M, Pappatella J, Pourcher V, et al. COVID-19 in a liver transplant recipient: Could iatrogenic immunosuppression have prevented severe pneumonia? A case report. World J Gastroenterol. 2020;26(44):7076-84.

51. Waisberg DR, Abdala E, Nacif LS, Haddad LB, Ducatti L, Santos VR, et al. Liver transplant recipients infected with SARS-CoV-2 in the early postoperative period: Lessons from a single center in the epicenter of the pandemic. Transpl Infect Dis. 2020:e13418.

52. Mathiasen VD, Oversoe SK, Ott P, Jensen-Fangel S, Leth S. Recovery of Moderate Coronavirus Disease 2019 in a Liver Transplant Recipient on Continued Immunosuppression: A Case Report. Transplant Proc. 2020;52(9):2703-6.

53. Kutzler HL, Poulos CM, Cheema F, O'Sullivan DM, Ali A, Ebcioglu Z, et al. COVID-19 in Solid Organ Transplant Recipients: Observations From Connecticut. Transplantation. 2021;105(1):e6-e8.

54. Mocchegiani F, Baroni GS, Vivarelli M. Mild impact of SARS-CoV-2 infection on the entire population of liver transplant recipients: the experience of an Italian Centre based in a high-risk area. Updates Surg. 2020;72(4):12913.

55. Goss MB, Galván NTN, Ruan W, Munoz FM, Brewer ED, O'Mahony CA, et al. The pediatric solid organ transplant experience with COVID-19: An initial multi-center, multiorgan case series. Pediatr Transplant. 2020:e13868. 
56. Imam A, Karatas C, Imam R, Armutlu A, Mecit N, Karakaya A, et al. Three Consequent Pediatric Liver Transplant Deaths in the COVID-19 Era. Int J Organ Transplant Med. 2020;11(4):202-6.

57. Heinz N, Griesemer A, Kinney J, Vittorio J, Lagana SM, Goldner D, et al. A case of an Infant with SARS-CoV-2 hepatitis early after liver transplantation. Pediatr Transplant. 2020;24(8):e13778.

58. Morand A, Roquelaure B, Colson P, Amrane S, Bosdure E, Raoult D, et al. Child with liver transplant recovers from COVID-19 infection. A case report. Arch Pediatr. 2020;27(5):275-6.

59. Lagana SM, De Michele S, Lee MJ, Emond JC, Griesemer AD, Tulin-Silver SA, et al. COVID-19 Associated Hepatitis Complicating Recent Living Donor Liver Transplantation. Arch Pathol Lab Med. 2020.

60. Ali Malekhosseini S, Nikoupour H, Gholami S, Shamsaeefar A, Arasteh P, Kazemi K, et al. A Report of 85 Cases of COVID-19 and Abdominal Transplantation From a Single Center: What Are the Associated Factors With Death Among Organ Transplantation Patients. Transplantation. 2021;105(1):90-9.

61. Belli LS, Duvoux C, Karam V, Adam R, Cuervas-Mons V, Pasulo L, et al. COVID-19 in liver transplant recipients: preliminary data from the ELITA/ELTR registry. Lancet Gastroenterol Hepatol. 2020;5(8):724-5.

62. Merli M, Perricone G, Lauterio A, Prosperi M, Travi G, Roselli E, et al. Coronaviruses and Immunosuppressed Patients: The Facts During the Third Epidemic. Liver Transpl. 2020;26(11):1543-4.

63. Rabiee A, Sadowski B, Adeniji N, Perumalswami PV, Nguyen $\mathrm{V}$, Moghe A, et al. Liver Injury in Liver Transplant Recipients With Coronavirus Disease 2019 (COVID-19): U.S. Multicenter Experience. Hepatology. 2020;72(6):1900-11.

64. Hussain A, Bhowmik B, do Vale Moreira NC. COVID-19 and diabetes: Knowledge in progress. Diabetes Res Clin Pract. 2020;162:108142.
65. Chu Y, Yang J, Shi J, Zhang P, Wang X. Obesity is associated with increased severity of disease in COVID-19 pneumonia: a systematic review and meta-analysis. Eur $\mathrm{J}$ Med Res. 2020;25(1):64.

66. British Transplantation Society, The Renal Association. Guidance on the management of transplant recipients diagnosed with or suspected of having COVID19 2020 [Available from: https://bts.org.uk/wp-content/ uploads/2020/07/Clinical-management-of-transplants-andimmunosuppression-updated-9th-July.pdf.

67. Colmenero J, Rodríguez-Perálvarez M, Salcedo M, AriasMilla A, Muñoz-Serrano A, Graus J, et al. Epidemiological pattern, incidence, and outcomes of COVID-19 in liver transplant patients. J Hepatol. 2021;74(1):148-55.

68. Fix OK, Hameed B, Fontana RJ, Kwok RM, McGuire BM, Mulligan DC, et al. Clinical Best Practice Advice for Hepatology and Liver Transplant Providers During the COVID-19 Pandemic: AASLD Expert Panel Consensus Statement. Hepatology. 2020;72(1):287-304.

69. Busauschina A, Schnuelle P, van der Woude FJ. Cyclosporine nephrotoxicity. Transplant Proc. 2004;36(2 Suppl):229S-33S.

70. Coll E, Fernández-Ruiz M, Sánchez-Álvarez JE, MartínezFernández JR, Crespo M, Gayoso J, et al. COVID-19 in transplant recipients: The Spanish experience. Am J Transplant. 2020.

71. Doná D, Torres Canizales J, Benetti E, Cananzi M, De Corti F, Calore E, et al. Pediatric transplantation in Europe during the COVID-19 pandemic: Early impact on activity and healthcare. Clin Transplant. 2020;34(10):e14063.

72. Mitrani RD, Dabas N, Goldberger JJ. COVID-19 cardiac injury: Implications for long-term surveillance and outcomes in survivors. Heart Rhythm. 2020;17(11):198490.

73. Shah W, Hillman T, Playford ED, Hishmeh L. Managing the long term effects of covid-19: summary of NICE, SIGN, and RCGP rapid guideline. BMJ. 2021;372:n136. 